\title{
Neutron Scattering Analysis of Water's Glass Transition and Micropore Collapse in Amorphous Solid Water
}

\author{
Catherine R. Hill, ${ }^{1}$ Christian Mitterdorfer, ${ }^{2}$ Tristan G. A. Youngs, ${ }^{3}$ Daniel T. Bowron, ${ }^{3}$ \\ Helen J. Fraser, ${ }^{1}$ and Thomas Loerting ${ }^{2, *}$ \\ ${ }^{1}$ Department of Physical Sciences, The Open University, Walton Hall, Milton Keynes MK7 6AA, United Kingdom \\ ${ }^{2}$ Institute of Physical Chemistry, University of Innsbruck, A-6020 Innsbruck, Austria \\ ${ }^{3}$ ISIS Facility, Rutherford Appleton Laboratory, Harwell Oxford, Didcot, Oxon OX11 OQX, United Kingdom
}

(Received 28 August 2015; published 26 May 2016)

\begin{abstract}
The question of the nature of water's glass transition has continued to be disputed over many years. Here we use slow heating scans $\left(0.4 \mathrm{~K} \mathrm{~min}^{-1}\right)$ of compact amorphous solid water deposited at $77 \mathrm{~K}$ and an analysis of the accompanying changes in the small-angle neutron scattering signal, to study mesoscale changes in the ice network topology. From the data we infer the onset of rotational diffusion at $115 \mathrm{~K}$, a sudden switchover from nondiffusive motion and enthalpy relaxation of the network at $<121 \mathrm{~K}$ to diffusive motion across sample grains and sudden pore collapse at $>121 \mathrm{~K}$, in excellent agreement with the glass transition onset deduced from heat capacity and dielectric measurements. This indicates that water's glass transition is linked with long-range transport of water molecules on the time scale of minutes and, thus, clarifies its nature. Furthermore, the slow heating rates combined with the high crystallization resistance of the amorphous sample allow us to identify the glass transition end point at $136 \mathrm{~K}$, which is well separated from the crystallization onset at $144 \mathrm{~K}$-in contrast to all earlier experiments in the field.
\end{abstract}

DOI: 10.1103/PhysRevLett.116.215501

Water's glass transition has remained controversial for decades [1]. A key question is whether or not the endothermic feature observed in calorimetry experiments upon heating amorphous ices indicates transition from the glassy state to the deeply supercooled liquid. McMillan and Los were the first to detect the subtle increase in water's heat capacity near $137 \mathrm{~K}$, which they assigned to the glass transition of water [2]. Historically, other researchers were unable to observe the same effect, either because it is so weak, or because it is obscured by enthalpy relaxation effects when unannealed samples are used [3]. In order to disentangle the exotherm caused by enthalpy relaxation from the increase in heat capacity caused by the glass transition, well-annealed amorphous ice samples have to be used. Typically, such samples are kept for $90 \mathrm{~min}$ at $129 \mathrm{~K}$ and then cooled back to $77 \mathrm{~K}$ prior to the calorimetry scan [4-6]. A glass-transition onset temperature $T_{g, \text { onset }}$ of $136 \pm$ $2 \mathrm{~K}$ was detected at standard heating rates of $30 \mathrm{~K} \mathrm{~min}^{-1}$ for a range of well-annealed amorphous ices, including (a) LDA (low-density amorphous ice) [6], (b) HGW (hyperquenched glassy water) [4], and (c) c-ASW (compact amorphous solid water) [5]. The glass transition in highdensity amorphous ice (HDA), has an onset temperature $T_{g, \text { onset }}$ of $116 \pm 2 \mathrm{~K}$ at ambient pressure, and has hence been called water's second glass transition [7].

Published by the American Physical Society under the terms of the Creative Commons Attribution 3.0 License. Further distribution of this work must maintain attribution to the author(s) and the published article's title, journal citation, and DOI.
In this work we deal exclusively with the nature of water's first, "traditional" glass transition. In LDA, c-ASW, and HGW ice "phases," the increase in heat capacity at the glass transition, $\Delta c_{p}$ gauged from endothermic changes in calorimetry scans, amounts to about $1 \mathrm{~J} \mathrm{~K}^{-1} \mathrm{~mol}^{-1}$, corresponding to one of the feeblest glass transitions ever detected. In addition, the whole width of the glass transition cannot be studied, because the exothermic phase change to crystalline ice terminates the glass transition before its end point can be observed [4-6,8,9].

For all these reasons, there has been significant speculation about the nature of the glass transition. The explanations fall into 5 broad areas: (i) a transition from the glassy to the liquid state involving diffusion [4-6], isotope exchange [10], and viscosity decrease [11], (ii) a transition from one glassy to another glassy state (of the ice), involving unlocking of re-orientational (torsional), but not translational diffusion and no change in viscosity [12], (iii) a pretransition, called the shadow glass transition [13,14], (iv) amorphous ice is crystal-like [15-17], or (v) it is an impurity effect [18].

Suggestions (i) and (iii) were tested by studying the heating and cooling rate dependence of water's glass transition [19]. The results were shown to be incompatible with scenario (iii) [19] and, consequently, suggestion (iii) was retracted [20]. Furthermore, these studies allowed us to show that ultraviscous water represents one of the strongest liquids known in terms of the Angell classification [20], consistent with Ref. [21]. Recently, the fragility index of water near $136 \mathrm{~K}$ was demonstrated to have the lowest value known for any liquid [7,22]; i.e., water is "superstrong" near $T_{g}$ [23]. 
The open question that remains to be addressed is whether diffusive, translational motion is involved in water's glass transition. We have used small angle neutron scattering to study c-ASW, which is slowly heated at $\sim 0.4 \mathrm{~K} \mathrm{~min}^{-1}$, and focus our attention on the structural changes associated with the collapse of the network of micropores in the ice as it warms. The advantage of this experimental technique over calorimetry experiments done in the past is that the competing endothermic and exothermic changes in the ice are not superimposed, so it allows unprecedented insight into the molecular processes occurring in the vicinity of $T_{q}$.

About $1 \mathrm{~g}$ of $\mathrm{D}_{2} \mathrm{O}$-c-ASW was produced by slow water vapor deposition onto a copper plate held at $77 \mathrm{~K}$. Dosing occurred in the line of sight mode (nonbaffled supersonic flow) as described in our earlier work [24]. The sample was characterized after recovery from the vacuum chamber, showing it was free of contamination with crystalline ice using x-ray diffraction and thermal analysis [24]. Nitrogen adsorption BET isotherms at $77 \mathrm{~K}$ show the pores to be micropores, i.e., of diameter $d \leq 21 \AA$ and total volume $\approx 0.17-0.21 \mathrm{~cm}^{3} \mathrm{~g}^{-1}$ [25]. Compared with the volume of pore-free ASW of $1.08 \mathrm{~cm}^{3} \mathrm{~g}^{-1}$ the total volume fraction of pores thus amounts to about $1 / 6-1 / 5$. The recovered samples were then stored under liquid $\mathrm{N}_{2}$, shipped (with cryogenic containment) to the NIMROD instrument at ISIS, and transferred into a null-scattering TiZr alloy cell, all under liquid $\mathrm{N}_{2}$, as described in Ref. [24]. The sample was then heated at $\approx 0.4 \mathrm{~K} \mathrm{~min}^{-1}$ from 77 to $144 \mathrm{~K}$, with four isothermal "pauses": of $20 \mathrm{~min}$ at $93 \mathrm{~K}, 35 \mathrm{~min}$ at $117 \mathrm{~K}, 55 \mathrm{~min}$ at $136 \mathrm{~K}$, and $68 \mathrm{~min}$ at $144 \mathrm{~K}$. According to calorimetric studies $T_{g \text {,onset }}$ is directly dependent on the heating rate, appearing at $124 \mathrm{~K}$ for heating rates of $0.17 \mathrm{~K} \mathrm{~min}^{-1}$ [8], $136 \mathrm{~K}$ for heating rates of $30 \mathrm{~K} \mathrm{~min}^{-1}$ [4-6], and $176 \mathrm{~K}$ for heating rates of $6 \times 10^{6} \mathrm{~K} \mathrm{~min}^{-1}$ [9]. Based on the rates used in our experiments, $T_{g, \text { onset }} \approx 124 \mathrm{~K}$.

Small angle neutron scattering is a nondestructive method particularly suitable for the study of granular or porous structures with dimensions between 10 and $1000 \AA$ [26]. The power of the near- and intermediate range order diffractometer (NIMROD) is that it allows simultaneous observation of the mesoscale and intra- and intermolecular bonding. The broad peaks centered at $1.7 \AA^{-1}$ in Figs. 1(a)-1(c) are indicative of c-ASW. Below $144 \mathrm{~K}$, no signs of crystallinity were detected at $Q>1 \AA^{-1}$, indicating that regardless of any other changes in the ice structure, the intermolecular bonding remains amorphous throughout. Crystallization was clearly detected a few minutes into the isothermal pause at $144 \mathrm{~K}$ [see narrowing at $1.7 \AA^{-1}$ and appearance of Bragg peaks in Fig. 1(d)]. The slope and the shape of the small angle neutron signal at $Q<1 \AA^{-1}$ can be used to investigate the properties of the ice on the mesoscale. It is evident from Figs. 1(a)-1(c) that the neutron scattering cross section is nonzero. Features common to our earlier work (and described in detail

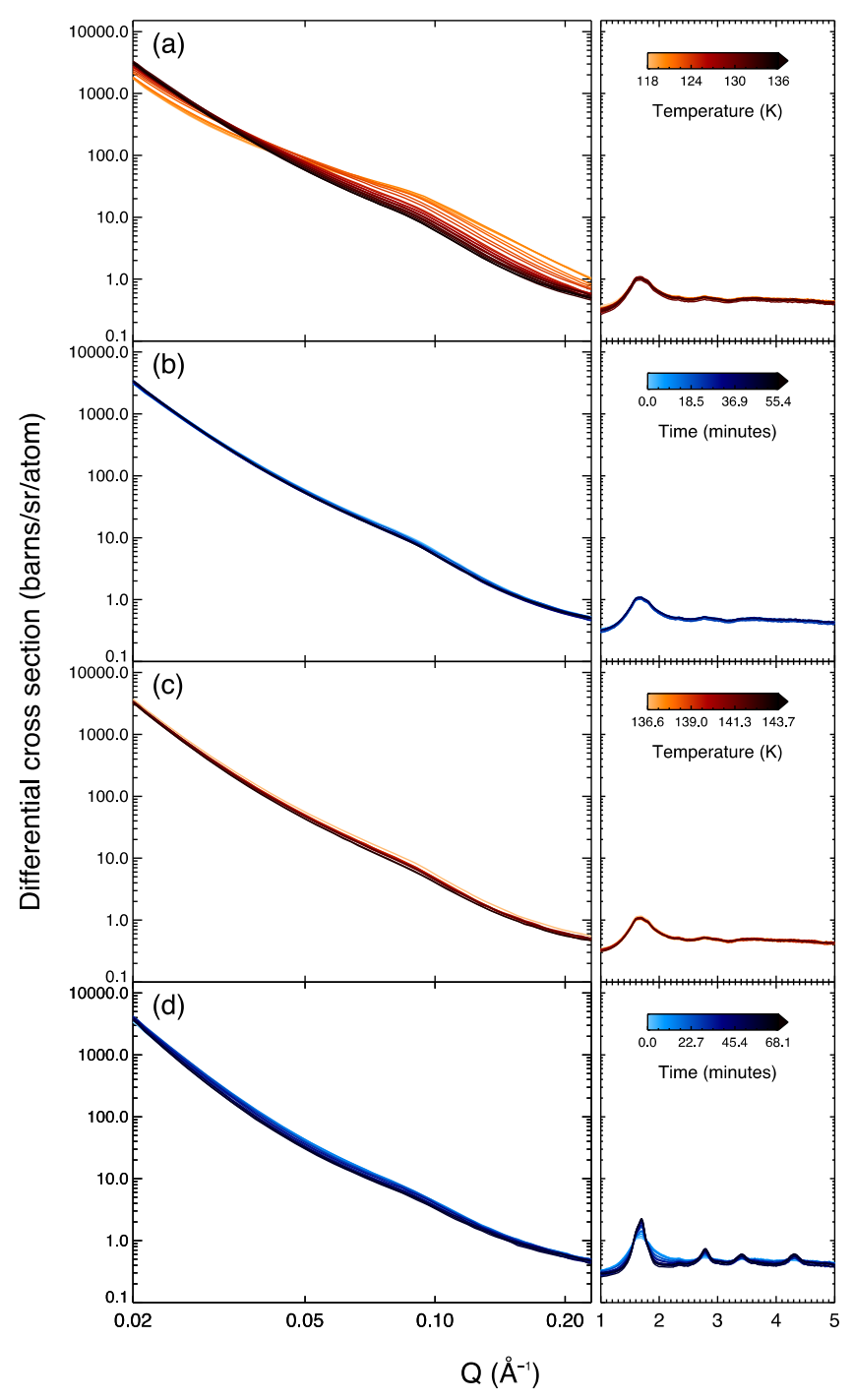

FIG. 1. Raw NIMROD neutron scattering data covering both the mesoscale and molecular scale scattering cross sections simultaneously. The evolution of the spectra is shown as a function of (a), (c) temperature, heating at $0.4 \mathrm{~K} \mathrm{~min}^{-1}$ and (b),(d) time, during an isothermal pause. The time-temperature evolution (in approximately $1 \mathrm{~K}$ steps or $5 \mathrm{~min}$ intervals) is indicated by the decreasing color scale of each plot.

therein) [24] indicate our c-ASW sample is both granular (the "slope") and porous (the "hump").

The raw data were analyzed using several approaches: First, we used a model-free approach to extract specific surface area (SSA) from the scattered intensity at small angles (see Ref. [24]). Standard linear plots $\mathrm{I}(Q) \times Q^{d}$ (with $d$ equal to 2.5 or 3.0) versus $Q$ show a "pseudopeak" at the distances corresponding to periodic pore spacings of $\approx 70 \AA$ [24]. These pseudopeaks can be seen clearly up to $120 \mathrm{~K}$, but then progressively disappear between 120 and $135 \mathrm{~K}$. Direct power-law fitting is used to extract the Porod exponent $\beta$ associated with the mass fractal dimension of the material. Second, we tried to fit the data using models. 
Whereas the Maxwell model assuming spherical pores fails, the Guinier-Porod (GP) model fits the data well [24]. The fit parameters are attributed to the pore shape ( $s$ parameter), pore-size (radius of gyration $R_{G}$ ), and nature of the interface between granules ( $d_{\mathrm{GP}}$ parameter). The outcomes of these analyses are shown in detail in Fig. 2. The parameters defining the ice structure can be broadly divided into three regimes - the temperature range prior to around $115 \mathrm{~K}$ where practically no changes occur, 115-144 K (the focus of the remainder of this Letter), and crystallization at $144 \mathrm{~K}$ and beyond.

The shape of the pores is never spherical, but much closer to cylindrical at $77-120 \mathrm{~K},(s \approx 1.5$ [Fig. 2(d)]). Beyond $121 \mathrm{~K}$ the pores progressively change shape: first they shrink along one axis and develop to 2D lamellae $(s=2)$ and then they disappear by $136 \mathrm{~K}$ ( $s$ approaches 3$)$. Even from visual inspection a drastic height loss of the ice sample can be noticed: the ice sample changes from rugged, with protrusions of about $1 \mathrm{~cm}$ in length, to smooth, with an overall thickness of a couple of $\mathrm{mm}$. Similar changes were monitored by optical interference measurements [27]. Not only the pore shape, but also $\beta$ [Fig. 2(b)] and $R_{G}$ [Fig. 2(e)] remain approximately constant at $77-120 \mathrm{~K}$, even during the two "isothermal pause" periods at $93 \mathrm{~K}$ and at $117 \mathrm{~K}$. In other words, the network of water molecules is translationally immobile both during heating and isothermal annealing on a time scale of hours at $<120 \mathrm{~K}$.

The SSA does not decrease at 80-120 K, but remains either constant or increases slightly with temperature. Both of the latter scenarios are consistent with the uncertainties related to the $\mathrm{I}(Q) \times Q^{4}$ analysis. One way to account for a SSA increase would be if pore clustering occurs in the ice, leading to larger (internal) pore surface areas in addition to the ice interface to the vacuum. Such a pore clustering was inferred by positron-annihilation spectroscopy [28] and recent kinetic Monte Carlo ice simulations [29]. Our c-ASW ice sample, however, exhibits no pore clustering - the pore shape, the radius $\left(R_{G}\right)$, and periodic spacings between pores all remain constant. Likewise, neither $d_{\mathrm{GP}}$ or $\beta$ are changing at $80-120 \mathrm{~K}$. Thus, the SSA either remains constant at $80-120 \mathrm{~K}$ or increases slightly due to the overall expansion of the bulk icy material. The main difference to other experiments [27,30-32] showing a continual decrease in SSA with temperature is their deposition mode for ASW samples, which is more akin to the "baffled" flow conditions used in our earlier work. Using baffled deposition we also saw the decrease of SSA with temperature [24]. The major difference between "nonbaffled, supersonic" flow conditions used here and baffled flow was explained in Ref. [33].

On the mesoscale our samples drastically change above $121 \mathrm{~K}$, and such changes occur prior to, and independently of, the amorphous to crystalline phase change. In all data, Figs. 2(a)-2(e), it is clear that significant changes are

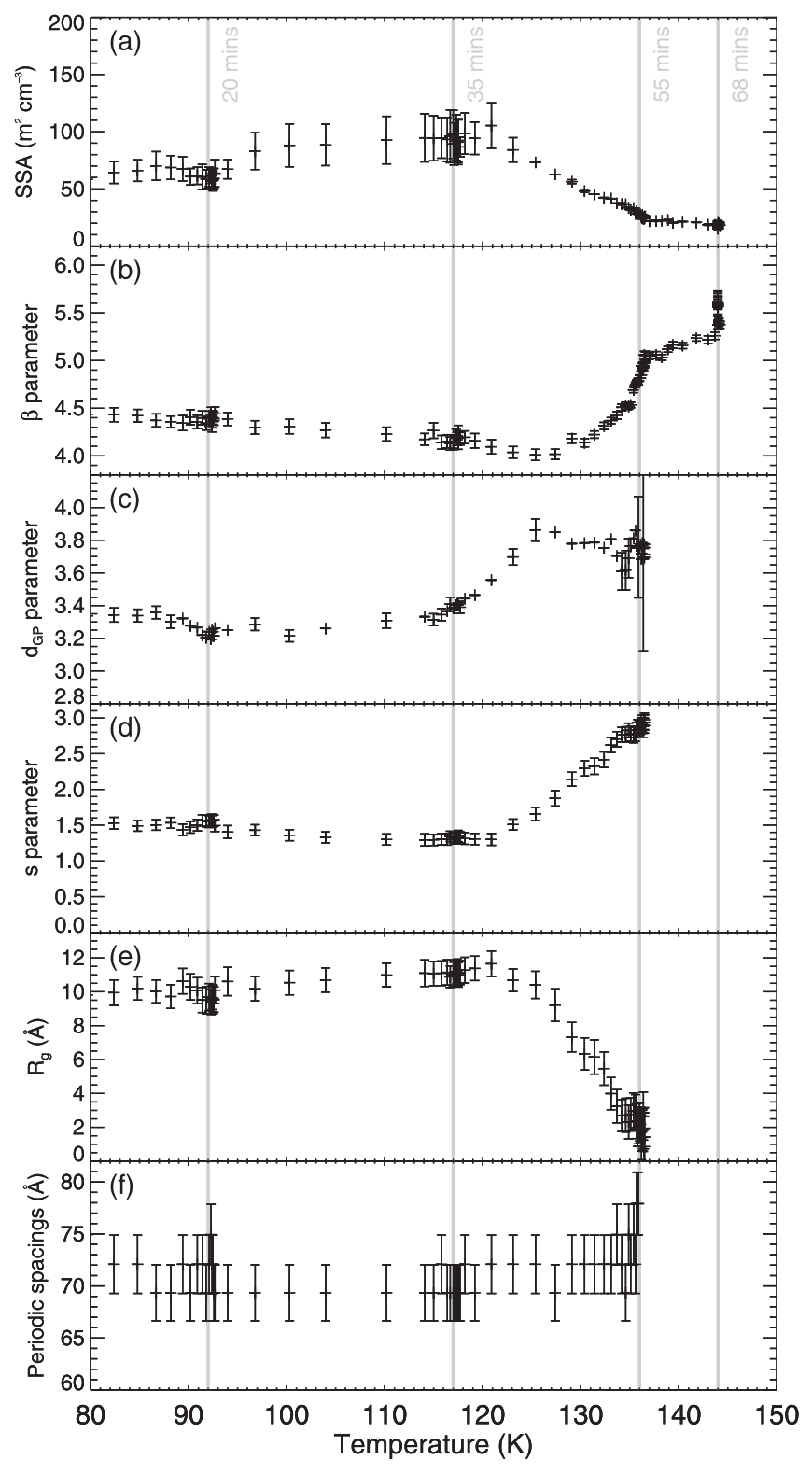

FIG. 2. Evolution of the key structural parameters derived from the neutron scattering differential cross section data with temperature. Isothermal stopping points are indicated by gray lines at $92,117,136$, and $144 \mathrm{~K}$, with the isothermal pause given alongside each line in minutes. (a) Specific surface area calculated from the plateau in $\mathrm{I}(Q) \times Q^{4}$ vs $Q$. (b) Porod $\beta$ parameter, determined from the low $Q$ region at $Q=$ $0.02-0.03 \AA^{-1}$ where $\mathrm{I}(Q)=D \times Q^{\beta}$, indicating the size, shape, and surface roughness of the granular scattering material. (c) Guinier-Porod $d$ parameter, calculated from the Guinier Porod fits to the data between around $Q=0.045-0.2 \AA^{-1}$, describing the internal surface roughness of the pores, where $d=3$ indicates a rough surface and $d=4$ indicates that the surface is smooth, on this length scale. (d) GP $s$ parameter, representing the geometry of the pores, where $s=0=$ sphere, $s=1=$ cylinder, $s=2=$ platelets, and $s \rightarrow 3$ as the pore collapses and disappears. (e) GP radius of gyration $R_{g}$ and (f) periodic spacing between pores, as calculated from in $\mathrm{I}(Q) \times Q^{d}$ vs $Q$ (where $d=2.5$ or 3 ). 
occurring during heating, particularly in the 121-136 K range: SSA and $R_{G}$ suddenly start to drop appreciably, and $s$ rises steeply. This indicates a new type of molecular motion governing the evolution of the mesoscale topology. This motion involves the collapse of the cylinderlike pores of $R_{G} \approx 11 \AA$ [Fig. 2(e)] and $d \leq 21 \AA$ [25]. Large-scale transport of water molecules over at least $10 \AA$ (pore radius) is necessary to achieve these kinds of changes. Assuming water self-diffusion and a random walk, the minimal selfdiffusion coefficient $D$ required to achieve these mesoscale changes can be estimated: $D>10^{-18} \mathrm{~cm}^{2} \mathrm{~s}^{-1}$ is required using a mean-square displacement $\left\langle x^{2}\right\rangle \approx 100 \AA^{2}$ and $t \approx 2500 \mathrm{sec}$ as the time it takes to complete the pore collapse between 120 and $136 \mathrm{~K}$. Consistent with this, Ghesquiére et al. calculate $D \approx 10^{-12} \mathrm{~cm}^{2} \mathrm{~s}^{-1}$ in this temperature range for a c-ASW sample based on MD trajectories on the $\mu$ s time scale, whereas $D$ is too small to be determined at lower temperatures [34]. We, therefore, attribute the observed structural changes to be a result of diffusive, translation motion. That is, from these data we infer the onset of molecular mobility (translational diffusion) and pore collapse occurs in our c-ASW sample at $121 \pm 1 \mathrm{~K}$. This is very close to the calorimetric $T_{g \text {,onset }}$ of $124 \mathrm{~K}$ [8]. At about the same temperature surface diffusion of a range of adsorbates [35] and isotopic mixing is initiated [10].

The Porod exponent $\beta$, and Guinier-Porod exponent $d_{\mathrm{GP}}$ indicate a third regime of change within the ice structure. From the start of the heating cycle $\beta$ continually decreases very slightly, reaching a minimum value at around $126 \mathrm{~K}$ before reversing direction and increasing again. $\beta$ is not related to the diffusive motion associated with pore collapse, nor changes in the SSA, but represents the nature of the interface between individual granules, as well as their shapes [24]. This parameter continues to change even in the isothermal waiting period at $144 \mathrm{~K}$, reflecting the sintering of ice granules as crystallization occurs. $d_{\mathrm{GP}}$ starts increasing at around $115 \mathrm{~K}$, which we interpret to reflect the onset of rotational (torsional) motion of the water molecules. Such motion leads to smoothing of the pore surfaces (reflected by an increase in $d_{\mathrm{GP}}$ ), without pore collapse occurring (no changes in $R_{G}$ or $s$ ). Pore collapse is accompanied by smoothing and shape changes in the ice grains (rapid increase in $\beta$ ), i.e., free molecular movement of $\mathrm{H}_{2} \mathrm{O}$. That is, c-ASW is in a liquid state above $T_{g}$. By $136 \mathrm{~K}$ the rapid changes in SSA and $\beta$ cease, and in the subsequent heating stage from 136-144 K change relatively little, indicating the end of the glass transition. The process is complete long before the ASW to $I_{c}$ transition at $144 \mathrm{~K}, 27 \mathrm{~min}$ into the isothermal step, which can be identified in both Figs. 1(d) and 2(b) (discontinuous jump in the $\beta$ parameter from 5.41 to 5.57). That is, there is a separation between the glass transition end point $T_{g \text {,end }}$ and the crystallization onset $T_{X}$. Such a separation was not found in earlier work on LDA-type samples. This is because in previous studies heating rates faster by about 2 orders of magnitude were employed [4-6]. In the case of the calorimetry study by Handa et al. [8] the heating rate is comparable, but they were using an LDA-I sample that started to crystallize at $133 \pm 1 \mathrm{~K}$, i.e., which is much less crystallization resistant than the c-ASW sample studied by us [6]. The width of the glass transition is thus $15 \mathrm{~K}$. For comparison, in earlier calorimetry work, a width of about $12 \mathrm{~K}$ was found between $T_{g \text {,onset }}$ and $T_{X}$ [4-6]. In other words, $T_{g \text {,end }}$ would appear just slightly above the crystallization onset in the calorimetry scans at $10-30 \mathrm{~K} \mathrm{~min}^{-1}$.

Thus, our data enable us to come back to the original purpose of our work-to describe the nature of water's glass transition, and distinguish between the five different hypotheses presented in the literature to explain the phenomenon. If just rotational (torsional) motion of water molecules at fixed oxygen positions, and/or point defect mobility similar to that known in crystalline ice was occurring near $T_{g, \text { onset }}$, as suggested in (ii) [12], then the pore collapse would not occur. Clearly this regime exists in the ice, between 115 and $121 \mathrm{~K}$, leading to pore surface smoothing, but not accompanied by a glass transition (or pore collapse), ruling out hypothesis (ii). The high $Q$ neutron scattering cross section data indicate an amorphous nature of the ice throughout the glass transition temperature range. The possibility of nanocrystalline ice [explanation (iv)] is ruled out by the low translational mobility typically seen in crystalline ice; i.e., pore collapse would not occur upon heating nanocrystalline ice. Previous work shows that ASW with "filled" micropores, covering a wide range of adsorbates and exposures, also experience pore collapse, like "pure" c-ASW [36]. Thus, impurities as put forward in explanation (v) are not driving the pore collapse. Instead it is driven from the reorientation of the hydrogen-bond network of water molecules itself [34]. The explanation of water's glass transition consistent with all the observations is (i) long-range diffusion brings c-ASW into an ultraviscous state, leading to mass-transport of water molecules in the sample. The increase in heat capacity seen in calorimetry experiments [8] and the time scale of about $100 \mathrm{sec}$ seen in dielectric relaxation spectra near $124 \mathrm{~K}$ [7] is, thus, associated with long-range translational diffusion of water molecules.

In conclusion, we have analyzed small-angle neutron scattering data in amorphous solid water. This analysis shows very subtle changes attributable to pore surface smoothing and cooperative, nontranslational motion of water molecules set in at $115 \mathrm{~K}$. Starting at $121 \mathrm{~K}$ the changes are much faster and reach completion at about $136 \mathrm{~K}$, i.e., within about $40 \mathrm{~min}$ at heating rates of $\approx 0.4 \mathrm{~K} \mathrm{~min}^{-1}$. This indicates that onset of rotational motion precedes onset of translational motion, and that $T_{g \text {,end }}$ is reached at $136 \mathrm{~K}$. This is notable because crystallization is only detected at $144 \mathrm{~K}$. Thus, in contrast to earlier investigations of water's glass transition 
[4-6,8,9], there is a good separation between $T_{g \text {,end }}$ and $T_{X}$, which allows us to determine the glass transition width to be $15 \mathrm{~K}$. These changes are associated with water molecules mobile enough to reach mean-square displacements $\left\langle x^{2}\right\rangle$ exceeding $100 \AA^{2}$, i.e., long-range, diffusive motion necessary to cause the collapse of the micropores and the $3 D \rightarrow 2 D$ topology change. The time scale extracted from the present work agrees with the time scale from calorimetry [4-6,8,9] and dielectric experiments [7] on wellannealed samples (after the network collapse). That is, the motion of water molecules at $>121 \mathrm{~K}$ in annealed, compact ASW samples takes place on the same time scale as the motion of water molecules causing the transition from porous ASW to compact ASW. Again, this is only possible if one assumes this motion is translational and diffusive in character. Furthermore, the effect is not a surface effect, but rather a bulk effect. The effect is also inconsistent with the idea of a crystal-like nature. The nature of water's glass transition, therefore, is glass to liquid, but not glass to glass or crystal-like to crystal-like. Our work also provides the relevant time scales to be investigated using methods probing dynamics directly rather than inferring the dynamics based on the mesoscopic structural changes as done here. The time scales of relevance can, for instance, be probed using broadband dielectric relaxation spectroscopy, spin echo ${ }^{2} \mathrm{H}$ NMR, or quasielastic neutron scattering, which will be the focus of future work.

Experiments at the ISIS Pulsed Neutron and Muon Source were supported by a beam time allocation from the Science and Technology Facilities Council (STFC). We gratefully acknowledge the ESF Short visit grant (MicroDICE) for C. M. The study is financed by a grant from the European Research Council (ERC Starting Grant SULIWA) and the Austrian Science Fund FWF (bilateral French-Austrian project I1392). C.R.H. and H. J.F. acknowledge support from The Open University. H. J.F. acknowledges the EU LASSIE FP7 ITN initiative (Laboratory Astrochemical Surface Science in Europe) Grant Agreement No. 238258 for her participation in this work. Astrochemistry at the Open University is supported by STFC under grant agreements No. ST/M007790/1, ST/ M003051/1, ST/N006488/1, ST/N005775/1 and ST/ L000776/1.

*thomas.loerting@uibk.ac.at

[1] S. Capaccioli and K. L. Ngai, J. Chem. Phys. 135, 104504 (2011)

[2] J. A. McMillan and S. C. Los, Nature (London) 206, 806 (1965).

[3] C. A. Angell, W. J. Sichina, and M. Oguni, J. Phys. Chem. 86, 998 (1982).

[4] G. P. Johari, A. Hallbrucker, and E. Mayer, Nature (London) 330, 552 (1987).
[5] A. Hallbrucker, E. Mayer, and G. P. Johari, Philos. Mag. B 60, 179 (1989).

[6] M. S. Elsaesser, K. Winkel, E. Mayer, and T. Loerting, Phys. Chem. Chem. Phys. 12, 708 (2010).

[7] K. Amann-Winkel, C. Gainaru, P. H. Handle, M. Seidl, H. Nelson, R. Böhmer, and T. Loerting, Proc. Natl. Acad. Sci. U. S. A. 110, 17720 (2013).

[8] Y. P. Handa and D. D. Klug, J. Phys. Chem. 92, 3323 (1988).

[9] A. Sepúlveda, E. Leon-Gutierrez, M. Gonzalez-Silveira, C. Rodríguez-Tinoco, M. T. Clavaguera-Mora, and J. Rodríguez-Viejo, J. Chem. Phys. 137, 244506 (2012).

[10] R. S. Smith and B. D. Kay, Nature (London) 398, 788 (1999).

[11] G. P. Johari, J. Phys. Chem. B 102, 4711 (1998).

[12] M. Fisher and J. P. Devlin, J. Phys. Chem. 99, 11584 (1995).

[13] Y. Yue and C. A. Angell, Nature (London) 427, 717 (2004).

[14] N. Giovambattista, C. A. Angell, F. Sciortino, and H. E. Stanley, Phys. Rev. Lett. 93, 047801 (2004).

[15] J. S. Tse, K. H. Tan, and J. M. Chen, Chem. Phys. Lett. 174, 603 (1990).

[16] H. Schober, M. M. Koza, A. Tolle, C. Masciovecchio, F. Sette, and F. Fujara, Phys. Rev. Lett. 85, 4100 (2000).

[17] V. P. Shpakov, P. M. Rodger, J. S. Tse, D. D. Klug, and V. R. Belosludov, Phys. Rev. Lett. 88, 155502 (2002).

[18] M. Chonde, M. Brindza, and V. Sadtchenko, J. Chem. Phys. 125, 094501 (2006).

[19] I. Kohl, L. Bachmann, E. Mayer, A. Hallbrucker, and T. Loerting, Nature (London) 435, E1 (2005).

[20] C. A. Angell, J. Phys. Condens. Matter 19, 205112 (2007).

[21] S. M. McClure, D. J. Safarik, T. M. Truskett, and C. B. Mullins, J. Phys. Chem. B 110, 11033 (2006).

[22] V. N. Novikov and A. P. Sokolov, Phys. Rev. Lett. 110, 065701 (2013).

[23] C. A. Angell, C. T. Moynihan, and M. Hemmati, J. Non-Cryst. Solids 274, 319 (2000).

[24] C. Mitterdorfer, M. Bauer, T. G. A. Youngs, D. T. Bowron, C. R. Hill, H. J. Fraser, J. L. Finney, and T. Loerting, Phys. Chem. Chem. Phys. 16, 16013 (2014).

[25] E. Mayer and R. Pletzer, Nature (London) 319, 298 (1986).

[26] A. K. Patra, S. Ramanathan, D. Sen, and S. Mazumder, J. Alloys Compd. 397, 300 (2005).

[27] J.-B. Bossa, K. Isokoski, M. S. Valois, and H. Linnartz, Astron. Astrophys. 545, A82 (2012).

[28] Y. C. Wu, J. Jiang, S. J. Wang, A. Kallis, and P. G. Coleman, Phys. Rev. B 84, 064123 (2011).

[29] S. Cazaux, J.-B. Bossa, H. Linnartz, and A. G. G. M. Tielens, Astron. Astrophys. 573, A16 (2015).

[30] H. J. Fraser, M. P. Collings, J. W. Dever, and M. R. S. McCoustra, Mon. Not. R. Astron. Soc. 353, 59 (2004).

[31] U. Raut, M. Famá, B. D. Teolis, and R. A. Baragiola, J. Chem. Phys. 127, 204713 (2007).

[32] R. S. Smith, N. G. Petrik, G. A. Kimmel, and B. D. Kay, Acc. Chem. Res. 45, 33 (2012).

[33] E. Mayer and R. Pletzer, J. Chem. Phys. 80, 2939 (1984).

[34] P. Ghesquière, T. Mineva, D. Talbi, P. Theulé, J. A. Noble, and T. Chiavassa, Phys. Chem. Chem. Phys. 17, 11455 (2015).

[35] F. Mispelaer, P. Theulé, H. Aouididi, J. Noble, F. Duvernay, G. Danger, P. Roubin, O. Morata, T. Hasegawa, and T. Chiavassa, Astron. Astrophys. 555, A13 (2013).

[36] R. A. Baragiola, in Water in Confining Geometries, edited by V. Buch and J. P. Devlin (Springer-Verlag, Heidelberg, 2003). 